\title{
Enthalpy Relaxation of Liquid Crystalline Polymer with Cyanobiphenyl Group in the Side Chain: Analysis based on Configurational Entropy Model with the AG Expression
}

\author{
By Yutaka TANAKA*
}

The enthalpy relaxation of poly(cyanobipheny hexylacrylate) has been studied by differential scanning calorimetry. The experimental results of the heat capacity $\left(c_{\mathrm{p}}\right)$ were compared with the prediction of a phenomenological model for the process of structural relaxation based on the configurational entropy $\left(S_{\mathrm{c}}\right)$ and the relaxation time expressed by the Adam-Gibbs equation. This model follows the evolution of the configurational entropy of the sample during the whole thermal history including an isothermal annealing. The parameters of the model were calculated by simultaneous fits to the experimental results. With the calculation result, the peak position appeared in DSC curve of $c_{\mathrm{p}}$ was interpreted using the plot of temperature dependence of $S_{\mathrm{c}}$. The plot showed that $S_{\mathrm{c}}$ rapidly approached to the configurational entropy of equilibrium $\left(S_{\mathrm{c}}{ }^{\mathrm{eq}}\right)$ in the narrow temperature range around $T_{\mathrm{g}}$, which is often referred to as the recovery of enthalpy, and thus the recovery of $S_{\mathrm{c}}$, in interpreting the enthalpy relaxation. As the measure of structural relaxation upon the isothermal ageing, the value of relaxation function $(\phi)$ was considered, which estimates the departure from the equilibrium. The correlation between $\phi$ of $S_{\mathrm{c}}$ and $\phi$ of enthalpy is also presented.

KEY WORDS: Liquid Crystalline Polymer / Cyanobiphenyl Group / DSC / Enthalpy Relaxation / Configurational Entropy / Adam-

Gibbs Theory /

The physical properties of the liquid crystalline polymer with cyanobiphenyl groups in the side chain (PCBA) have been studied with respect to the effects of molecular weight and spacer length. ${ }^{1,2}$ In the previous paper, the enthalpy relaxation measured using Differential Scanning Calorimetry (DSC) was analysed with the activation energy spectrum (AES) model. ${ }^{3}$ This model provides information about thermally activated processes which are available to contribute to observed changes in the properties upon physical ageing. For the enthalpy relaxation of PCBA6 (PCBA in which the spacer length is 6), the analysis gives the relation between the process number and the activation energy, in other words, the distribution of the relaxation process.

In general, the decrease in enthalpy upon the isothermal ageing at temperature below the glass transition temperature $T_{\mathrm{g}}$, is closely related to the structural relaxation toward equilibrium in the glassy state. Concerning the relaxation of PCBA6, the optical texture was shown to vary in the previous paper, as an evidence of structural change. The change in optical texture is being considered as the coarsening dynamics of the disclinations. That is to say, the dynamics of PCBA6 molecules bring about the change in structure.

On the other hand, the modelling approaches for enthalpy relaxation of polymer have been reported, which attempts to reproduce the measured DSC curve using quantities of state. The quantities of state employed are the configurational entropy, $S_{\mathrm{c}}$, and the fictive temperature, $T_{\mathrm{f}}{ }^{4,5}$ It has been shown that the main features of the relaxation process can be modelled on the basis of a distribution of relaxation times that depends both on the temperature and on the structure of the material represented by the value of the relaxing variable, that is $\tau=\tau\left(T_{\mathrm{f}}, T\right)$.

The modelling approach for the enthalpy relaxation of polymer was first attempted by Hodge and coworkers. ${ }^{6,7}$ They succeeded to reproduce DSC curves of normalised $c_{\mathrm{p}}$ using a mathematical model containing $\tau$ expressed as a function of $T_{\mathrm{f}}$. Afterwards, another model was proposed in reproducing DSC curve on the basis of the configurational entropy; here this is called as SC model. ${ }^{8-13}$ The basic feature which is common to both models is the application of Boltzmann superposition principle, which enables to calculate the quantities of the state corresponding to the thermal history the polymer had experienced.

These two models currently accompany several variations to predict DSC results accurately.,14-16 The arguments are taken on the equation to define $\tau$. The equation accepted widely at first was a generalised Arrhenius law, ${ }^{17}$

$$
\tau=A \exp \left(\frac{x \Delta h}{R T}+\frac{(1-x) \Delta h}{R T_{\mathrm{f}}}\right)
$$

where $x(0<x<1)$ is the non-linearity parameter partitioning the structural and thermodynamic contribution to $\tau$. $\Delta h$ is the activation energy, $R$ gas constant, $A$ pre-exponential factor. Further, entropy-based theories have been tested for the 
equation of $\tau$. The Adam-Gibbs equation (AG) was adopted to give the equation of $\tau$ as;

$$
\tau=A \exp \left(\frac{B}{T S_{c}}\right)
$$

where $B$ is a constant parameter. $S_{\mathrm{c}}$, the configurational entropy will be refereed to later as $S_{\mathrm{c}}(t)$ taking account that $S_{\mathrm{c}}$ is a function of experimental time, $t$. Also the equation containing the well-established Vogel expression can be seen.

$$
\tau=A \exp \left(\frac{B}{R T\left(1-T_{2} / T_{\mathrm{f}}\right)}\right)
$$

In addition to these three, other forms have been proposed to investigate their quality.

The investigation whether each of these $\tau$-expressions fits to the results of PCBA6 or not is outside of this study. The scope of this study is to introduce an accurate measure to the change of structure as a result of molecular dynamics. As a first step, this paper shows that the value of relaxation function $(\phi)$ has the meaning of the measure.

The reason to introduce SC model into the DSC result of this study instead of the model of fictive temperature was described here. At first, both of them can measure the structural state, whereas the configurational entropy is more likely to be accepted as a general quantity of state. Second, in the calculation of the configurational entropy, $\Delta c_{\mathrm{p}}$ obtained by the experiment is taken into consideration. While, the calculation of the fictive temperature does not contain $\Delta c_{\mathrm{p}}$ nor the experimental value. Third, the calculation of the fictive temperature sometimes takes an Arrhenius temperature dependence of $\tau$ to result in unusually large values of $\Delta h / R$ in eq $1 .^{18}$

To introduce the measure to the structural relaxation of PCBA is of interest from several points of view. (1) It estimates the change of optical texture by the thermal ageing. (2) It estimates the effect of the spacer length on the change of structure. (3) It has the close relationship with AES curve.

As for (1), the correlation function is currently used to analyse the coarsening dynamics. ${ }^{19}$ The analysis with SC model can possibly be the alternative method to investigate it.

As for (2), although the analyses for PCBA2 and PCBA11 are not completed, SC model is expected to clarify the role of the spacer chain. It is predicted at first sight that the configurational entropy will increase as the increase in the spacer length.

As for (3), AES model and SC model are entirely different and in conflict with each other in some places. For example, $\tau$ takes only one value at a given ageing temperature in AES model, while it varies during the isothermal ageing in SC model. Further, the pre-exponential factor relates to Debye temperature in AES model, whereas it is determined independently of Debye temperature in SC model. Nevertheless. it has significance to investigate results obtained from two different analysis methods. Some expanded ideas help to solve the confliction; for example, $\tau$ of AES model is apparently a representative of several relaxation times during the thermal ageing.
With these backgrounds this paper is concerned with the result of the analysis with $\mathrm{SC}$ model including AG equation on $c_{\mathrm{p}}(T)$ curve of PCBA6 obtained previously by DSC.

\section{ANALYSIS}

The brief description concerning SC model shown in this paper was given for physical quantities and variables used in the analysis of this study. More equations and variables, and hence matters to be attended to, appear in the whole theory for the model to reproduce DSC curve (see ref 7-9,15 and references cited therein), where the Tool-NarayanaswamyMoynihan approach is the basic grounds. ${ }^{20,21}$

The non-exponentiality of the relaxation can be written by the stretched exponential form with the shape parameter of $\beta$ $(0<\beta \leq 1){ }^{22}$

$$
\phi=\exp \left[-\left(\frac{t}{\tau}\right)^{\beta}\right]
$$

$\beta$ describes the broadness of the spectrum of relaxation times.

The configurational entropy at a time $t, S_{\mathrm{c}}(t)$ is regarded as a function of the entire temperature history the polymer sample had carried on up to $t$ and $T(t)$. The relaxation time which characterise the relaxation process depends both on the temperature and on the structural state represented by $S_{\mathrm{c}}(t)$; $\tau=\tau\left[S_{\mathrm{c}}(t), T(t)\right]$. This function is assumed to have the same form as Adam and Gibbs' equation of eq 2, which was originally intended by the authors only for equilibrium. ${ }^{23}$ In fact, if the configurational entropy in eq 2 has the equilibrium value $S_{\mathrm{c}}{ }^{\mathrm{eq}}(T)$, it gives the equation of;

$$
\tau\left[S_{\mathrm{c}}{ }^{\mathrm{eq}}(T), T\right]=A \exp \left(\frac{B}{S^{\mathrm{eq}}(T) \cdot T}\right)=\tau^{\mathrm{eq}}(T)
$$

which defines Adam and Gibbs' curve of the equilibrium relaxation time, $\tau^{\mathrm{eq}}(T)$. The parameter $B$ in Adam and Gibbs' theory is related to the microscopic picture of the process through $B=s_{\mathrm{c}} \Delta \mu / k$, where $\Delta \mu$ is the free energy barrier hindering configurational rearrangement per mol of molecules or chain segments, and $k$ Boltzmann's constant. $s_{\mathrm{c}}$ means the configurational entropy of the smallest cooperatively rearranging region, which is analogous to the enthalpy change, $c(E)$ in AES model.

Further, SC model includes an assumption that the limiting value exists for $S_{\mathrm{c}}(t)$. This is the asymptotic value of $S_{\mathrm{c}}(t)$ when $t \rightarrow \infty$; it is determined uniquely for a given temperature and is independent of further details of the temperature history. For this limit state at in finite time $S_{\mathrm{c}} \lim (T)$ can be given. Then, $S_{\mathrm{c}}(t)$ is expressed as;

$$
\begin{aligned}
S_{\mathrm{c}}(t)= & S_{\mathrm{c}}^{\lim }(T)-\sum_{i=1}^{n}\left[S_{\mathrm{c}}^{\lim }\left(T_{i}\right)-S_{\mathrm{c}}^{\lim }\left(T_{i-1}\right)\right] \\
& \times \exp \left[-\left(\sum_{k=i}^{n} \frac{\Delta t_{k}}{\tau_{k}}\right)^{\beta}\right]
\end{aligned}
$$

where, $t=\sum_{k=1}^{n} \Delta t_{k}$. In deriving eq 6 , Ribelles et al. employ reduced time ${ }^{24}$ to write the elapsed time $t .{ }^{8,9}$ However, the 
expression of summation was shown here in order to emphasise the practical equation used in the calculation.

If the limit state of the structural relaxation process would coincide with the extrapolation of the equilibrium values determined at temperatures above the glass transition, $S_{\mathrm{c}}{ }^{\lim }(T)=S_{\mathrm{c}}{ }^{\mathrm{eq}}(T)$ could be given with,

$$
S_{\mathrm{c}}^{\mathrm{eq}}(T)=\int_{T_{2}}^{T} \frac{\Delta c_{p}(\theta)}{\theta} d \theta
$$

where $\Delta c_{\mathrm{p}}(T)=c_{\mathrm{pl}}(T)-c_{\mathrm{pg}}(T)$ is the configurational heat capacity, the difference in the heat capacity between equilibrium liquid and glass at the same $T . T_{2}$ is the Gibbs-DiMarzio transition temperature, ${ }^{25}$ note that $T_{2}$ should be distinguished from the second term of the sequence $T_{i}$. Further, the limiting value of $\Delta c_{\mathrm{p}}(T)$ can be written as $\Delta c_{\mathrm{p}}^{\lim }(T)=c_{\mathrm{p}}{ }^{\lim }(T)-$ $c_{\mathrm{pg}}(T)$ and,

$$
\Delta c_{\mathrm{p}}^{\lim }(T)=T \frac{d S_{c}^{\lim }(T)}{d T}
$$

$S_{\mathrm{c}}{ }^{\lim }(T)$ is calculated as

$$
S_{\mathrm{c}}{ }^{\lim }(T)=S_{\mathrm{c}}{ }^{\mathrm{eq}}\left(T^{*}\right)+\int_{T^{*}}^{T} \frac{\Delta c_{p}^{\lim }(\theta)}{\theta} d \theta .
$$

In eq $9, T^{*}$ is a temperature above $T_{\mathrm{g}}$ in the equilibrium liquid state, where the limit state coincides with the equilibrium state. $T^{*}=T_{\mathrm{g}}+10^{\circ} \mathrm{C}$ was taken in this study.

The application of the set of these equations to the thermal history leads to the expression of $S_{\mathrm{c}}(t)$.

$$
\begin{aligned}
S_{\mathrm{c}}(t)= & S_{\mathrm{c}}{ }^{\lim }[T(\mathrm{t})]-\sum_{i=1}^{n}\left(\int_{T_{i-1}}^{T_{i}} \frac{\Delta c_{p}^{\lim }(\theta)}{\theta} d \theta\right) \\
& \times \exp \left[-\left(\sum_{k=i}^{n} \frac{\Delta t_{k}}{\tau_{k}}\right)^{\beta}\right]
\end{aligned}
$$

The resulting equation has four adjustable parameters: $A, B, T_{2}$ and $\beta$. Three of them, $A, B, T_{2}$, together with $\Delta c_{\mathrm{p}}{ }^{\lim }(T)$ define the dependence of the equilibrium relaxation times from eq 5 .

Eq 10 must be solved numerically. In this work the cooling and heating in the thermal histories were replaced by a series of 0.5 degree temperature jumps followed by isothermal stages with a duration calculated to result in the same overall rate of temperature change as in the actual experiments. For the specified temperature the configurational entropy was calculated with eq 10 , then the relaxation time corresponding to that configurational entropy was calculated using eq 2 .

The analysis requires to express the experimental results and calculated values in terms of $\Delta c_{\mathrm{p}}(T)$ curves, whereas there is no straightforward relationship between the configurational entropy and $c_{\mathrm{p}}(T)$. However, the following relation can be used;

$$
c_{\mathrm{p}}(T)-c_{\mathrm{pg}}(T)=\frac{\partial H_{\mathrm{c}}}{\partial T}
$$

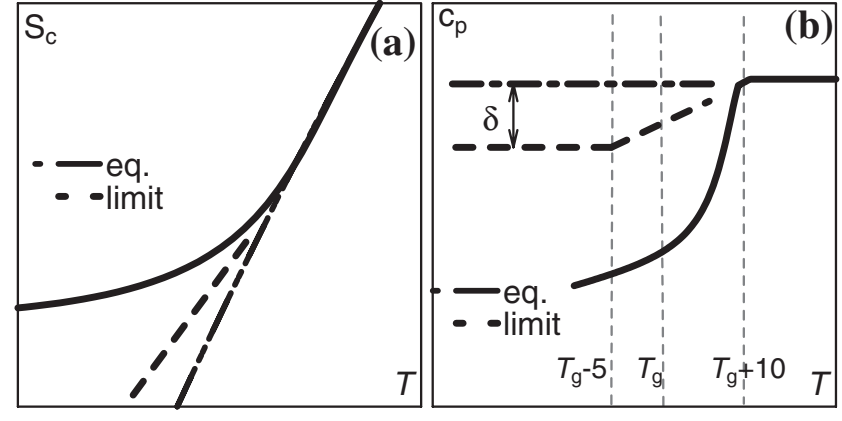

Figure 1. (a) Schematic representation of the configurational entropy corresponding to the equilibrium state (dashed-dotted-line), to the hypothetical line of the limit state of the structural relaxation process (dashed line), and to an experimental cooling scan at a finite cooling rate (solid line). (b) $c_{p}(T)$ curves corresponding to the three cases of (a). The phenomenological parameter $\delta$ introduced in ref 8 represents the shift in the limit glassy heat capacity (dashed line) with respect to its extrapolation from the equilibrium state (dashed-dotted-line).

where $H_{\mathrm{c}}$ is the specific configurational enthalpy. For the dependence of $H_{\mathrm{c}}$ on the thermal history an analogous manipulation to $S_{\mathrm{c}}(t)$ is;

$$
\begin{aligned}
H_{\mathrm{c}}(t)= & H_{\mathrm{c}}^{\lim }[T(\mathrm{t})]-\sum_{i=1}^{n}\left[H_{\mathrm{c}}^{\lim }\left(T_{i}\right)-H_{\mathrm{c}}^{\lim }\left(T_{i-1}\right)\right] \\
& \times \exp \left[-\left(\sum_{k=i}^{n} \frac{\Delta t_{k}}{\tau_{k}}\right)^{\beta}\right] .
\end{aligned}
$$

The assumption made here in order to determine $H_{\mathrm{c}}$ is that enthalpy and entropy have the same relaxation function $\phi$; in other words, the relaxation times for enthalpy and entropy are same. $c_{\mathrm{p}}(T)$ evaluated using eq 11 was compared with the experimental result.

It is necessary to make an additional assumption on $c_{\mathrm{p}}{ }^{\lim }(T)$ because we have no information concerning the temperature dependence of $c_{\mathrm{p}}{ }^{\lim }(T)$. Its phenomenological shift with respect to $c_{\mathrm{pl}}(T)$ was taken into consideration in a narrow temperature range around $T_{\mathrm{g}}$ to account for the overestimation of the enthalpy of polymer appeared in the model of ToolNarayanaswamy-Moynihan. The phenomenological shift has been widely accepted in applying SC model. .,10-14 $^{\text {S }}$

The behaviour of the configurational entropy was schematically represented in Figure 1 for the liquid state (equilibrium), for an experimental scan at finite rate (solid line), and for the hypothetical limit of the structural relaxation process. The simplest shape is assumed for $S_{\mathrm{c}} \lim$ (see Figure 1), which leads to a constant shift parameter $\delta$ for the heat capacity. SC model usually includes the $\delta$ parameter.

\section{EXPERIMENTAL}

The polymer under study is side-chain liquid crystalline polyacrylate containing cyanobiphenyl mesogenic units connected by hexamethylene spacers to the main chain; which is written by PCBA6. Preparation and characterisation procedure were described as sample code of $\mathrm{B}$, in the previous paper. ${ }^{3}$ 
Table I. Results of isothermal ageing experiments for PCBA6. $T_{\mathrm{A}}$ is the ageing time. $\boldsymbol{\beta}$ and $\boldsymbol{\tau}$ are parameters of eq 4

\begin{tabular}{ccr}
\hline$T_{\mathrm{A}} / \mathrm{K}$ & $\boldsymbol{\beta}$ & $\boldsymbol{\tau} / \mathrm{min}$ \\
\hline 291 & 0.487 & 17900 \\
296 & 0.433 & 6020 \\
301 & 0.414 & 1100 \\
306 & 0.336 & 205 \\
\hline
\end{tabular}

The value of $T_{\mathrm{g}}$ was determined according to the appearance of enthalpy loss. The molecular weight was determined by GPC calibrated with polystyrene standards. PCBA6 has $M_{\mathrm{n}}=1.1 \times$ $10^{5}$ and $M_{\mathrm{w}}=2.0 \times 10^{5}$; the glass transition and the clearing temperatures are $T_{\mathrm{g}}=312 \mathrm{~K}$ and $T_{\mathrm{cl}}=402 \mathrm{~K}$ respectively. The phase behaviour was observed under a polarising optical microscope equipped with a hot stage and confirmed to be the same as that reported previously. ${ }^{26}$

$c_{\mathrm{p}}(T)$ curves of PCBA6 were obtained from the DSC curves of $\mathrm{d} q / \mathrm{d} t v s$. time. ${ }^{27}$ The calibration was carried out with reference to $c_{\mathrm{p}}$ of Indium of $250 \mathrm{~K}, 298 \mathrm{~K}$ and $400 \mathrm{~K}$. In the measurements, the sample was (i) first maintained at $433 \mathrm{~K}$, well above $T_{\mathrm{cl}}$ for $5 \mathrm{~min}$ to erase any previous thermal history; (ii) cooled to a specified ageing temperature, $T_{\mathrm{A}}$ with a cooling rate of $12 \mathrm{~K} / \mathrm{min}$; (iii) annealed for the ageing period $t_{\mathrm{A}}$; and (iv) cooled to a temperature well below $T_{\mathrm{g}}\left(T_{\mathrm{g}}-50 \mathrm{~K}\right)$; then the DSC curve was recorded on heating with a rate of $5 \mathrm{~K} / \mathrm{min}$. Before each measurement, the reference scan was recorded following the steps (i), (ii) and (iv).

Previously obtained experimental results of $T_{\mathrm{A}}, \tau$ and $\beta$ were given in Table I. $c_{\mathrm{p}}(T)$ of PCBA6 was fitted to the linear equation of;

$$
c_{\mathrm{p}}(T)=a T+b .
$$

The coefficients for $T_{\mathrm{g}} \ll T$ was determined as $a=-2.56 \times$ $10^{-4}, \quad b=1.32$, and for $T \ll T_{\mathrm{g}}$ as $a=-19.3 \times 10^{-4}$, $b=0.283$, then used to calculate $S_{\mathrm{c}}{ }^{\text {eq }}(T)$ using eq 7 .

\section{RESULTS AND DISCUSSION}

SC model contains adjustable parameters of $A, B, T_{2}, \beta$ and $\delta$. To find these five parameters, the assumption of $S_{\mathrm{c}}{ }^{\lim }=S_{\mathrm{c}}{ }^{\text {eq }}$ was first made after the manner shown by Ribelles et al., i.e., $\delta=0 .{ }^{10}$ Second, experimentally determined $\beta$ shown in Table I were applied to eqs 10 and 12 . Then $A, B, T_{2}$ were determined with the search method. Although $\beta$ shown in Table I was derived with the constant relaxation time during the ageing, which is controversial as mentioned in the introduction section, the values were used by attaching importance to the experimental results. In fact, it is reported that the value of $\beta$ does not depend very well on the choice of other parameters. ${ }^{16}$ Therefore it is acceptable that $\beta$ was fixed with choosing the experimentally determined value before the fitting procedure.

The correlation between parameters has been amply reported for phenomenological models such as those of Tool-Narayanaswamy-Moynihan or Schere-Hodge, ${ }^{7,28-31}$ and

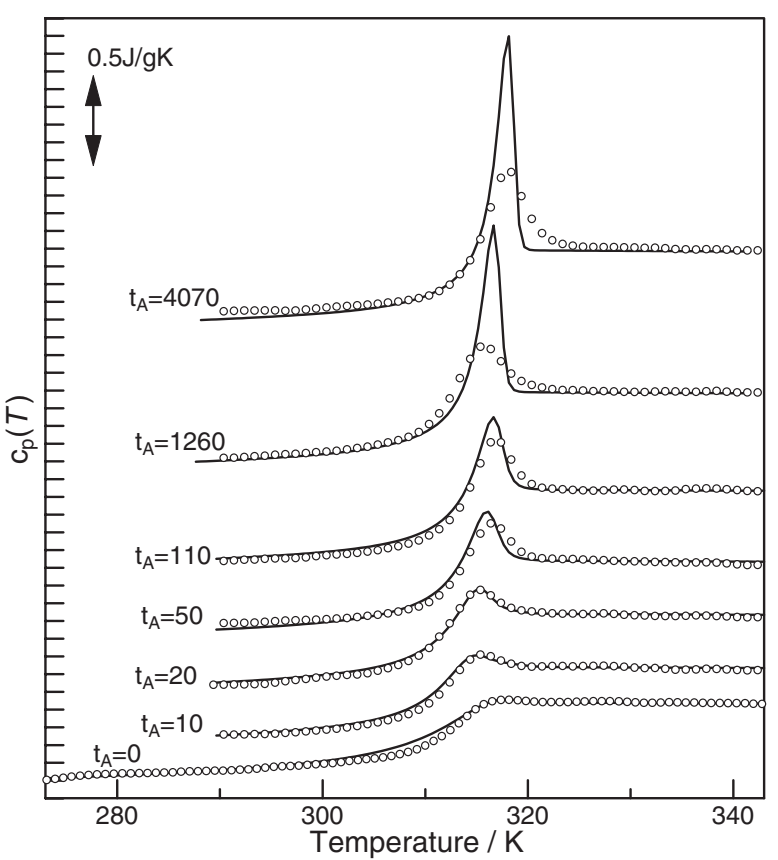

Figure 2. Experimental $c_{\mathrm{p}}(T)$ curves (indicated by open circles) of PCBA6 measured with $T_{\mathrm{A}}=306 \mathrm{~K}$ and different ageing times $\left(t_{\mathrm{A}}\right.$, the unit is $\min$.). $t_{A}=0$ denotes the reference scan. The solid lines represents the calculation curves with the assumption of $S_{\mathrm{C}}{ }^{\lim }(T)=S_{\mathrm{c}}{ }^{\mathrm{eq}}(T), B=1500 \mathrm{~J} / \mathrm{g}$ and the remaining parameters are shown in Table II.

Table II. Parameters of the model found with the assumption $S_{c} \lim (T)=S_{c}{ }^{e q}(T)$ for different choices of $B$

\begin{tabular}{ccc}
\hline$B / \mathrm{Jg}^{-1}$ & $T_{2} /{ }^{\circ} \mathrm{C}$ & $\ln (A / \mathrm{min})$ \\
\hline 1000 & -10 & -44 \\
1500 & -18 & -57 \\
2000 & -21 & -63 \\
2500 & -26 & -76 \\
3000 & -32 & -84 \\
\hline
\end{tabular}

was also found in the present work. Thus, several different sets of three parameters lead to nearly identical model predictions of $c_{\mathrm{p}}(T)$ curves for a given thermal history. As a basic propensity, a correlation was found between $B$ and $T_{2}$. To solve this problem, the parameter $B$ was kept fixed while $A$ and $T_{2}$ were adjusted, the fitting procedure was then carried out for different values of $B$. The procedure thus leads to a set of parameters of $A$ and $T_{2}$, for each fixed value of $B$.

Figure 2 shows the experimental $c_{\mathrm{p}}(T)$ results carried out for $T_{\mathrm{A}}=306 \mathrm{~K}$ and the fit obtained keeping $B=1500 \mathrm{Jg}^{-1}$ constant. The values obtained for $A$ and $T_{2}$ with the search method were shown in Table II. The parameters with different $B$ were found to be different from one set to another. The model was unable to reproduce accurately all the $c_{\mathrm{p}}(T)$ curves experimentally obtained for $T_{\mathrm{A}}=306 \mathrm{~K}$ with a single set of parameters.

The difference in peak heights of the overshoot between the experimental result and the calculation curve became more significant as the increase in $t_{\mathrm{A}}$. In particular, when $t_{\mathrm{A}}$ longer than $1000 \mathrm{~min}$, the peak height of the calculation curve was 


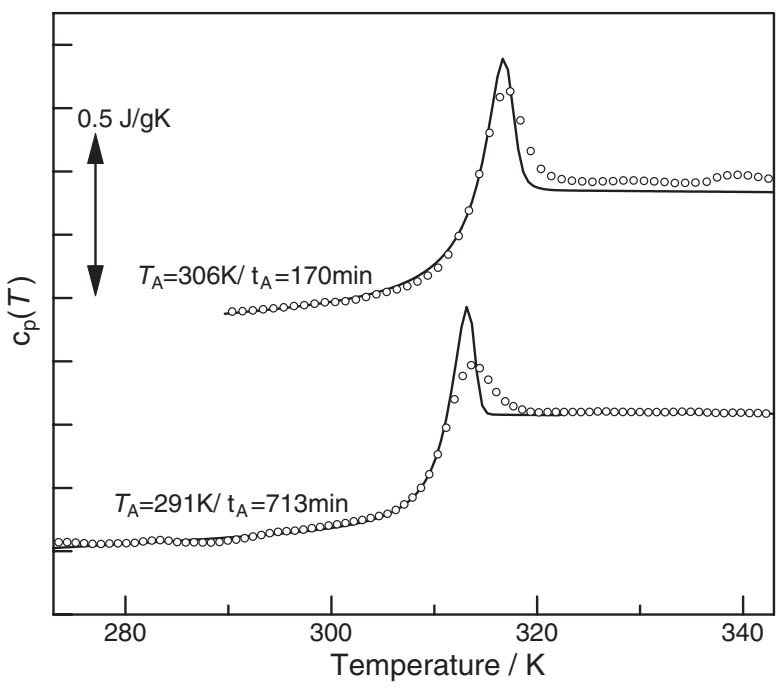

Figure 3. Experimental $c_{\mathrm{p}}(T)$ curves (indicated by open circles) of PCBA6 measured with different isothermal ageings. The solid lines represents the calculation curves with the assumption of $S_{\mathrm{c}}{ }^{\lim }(T)=S_{\mathrm{c}}{ }^{\mathrm{eq}}(T), B=1500 \mathrm{~J} / \mathrm{g}$ and the remaining parameters are shown in Table II.

much higher than the height of the experiment. This behaviour was observed for all the selected values of the parameter $B$. Further, concerning the experimental results of other $T_{\mathrm{A}}$, the differences between the experiment and the calculation curve were significant for $t_{\mathrm{A}}$ longer than $1000 \mathrm{~min}$.

However, for shorter time of $t_{\mathrm{A}}$, the calculation curve overall reproduced the experimental $c_{\mathrm{p}}(T)$ well. The result that the extent of reproducibility depended on $t_{\mathrm{A}}$ is considered to have little relationship with the departure from the equilibrium state, because $\tau$ varies according to $T_{\mathrm{A}}$ as shown in Table I.

The position of the endothermic peak experimentally observed, slightly shifted to higher temperature side with the ageing time. The position shift was also observed by the rise in $T_{\mathrm{A}}$ as shown in Figure 3 , where $T_{\mathrm{A}}=306 \mathrm{~K}$ is the highest ageing temperature in the experiment and $T_{\mathrm{A}}=291 \mathrm{~K} \approx$ $T_{\mathrm{g}}-20$, the lowest temperature. These results shows that the peak position is influenced by both the ageing temperature and time. In addition, $c_{\mathrm{p}}(T)$ peaks appeared at temperatures above $T_{\mathrm{g}}$ in all the measured DSC curves, as happens in other polymers of poly(methylmethacrylate), ${ }^{28}$ poly(vinyl chloride) ${ }^{30}$ polystyrene ${ }^{31}$ and poly $\left(\gamma\right.$-benzyl-L-glutamate). ${ }^{32}$

The peak position has relationship with the process of recovery of enthalpy, and thus recovery of entropy, during the heating of the aged sample. Further, the relationship with the molecular mobility of the chain segments has been pointed out concerning the recovery of enthalpy. ${ }^{33}$ In addition, the peak position is accounted for by the evolution of the out-ofequilibrium configurational entropy; in other words, it is $S_{\mathrm{c}}(t)$ evaluated for the heating scan which follows the isothermal ageing. ${ }^{34}$

In order to verify the relationship between the peak position and $S_{\mathrm{c}}(t)$, those obtained by the calculations for different thermal treatments were plotted in Figure 4; some data in

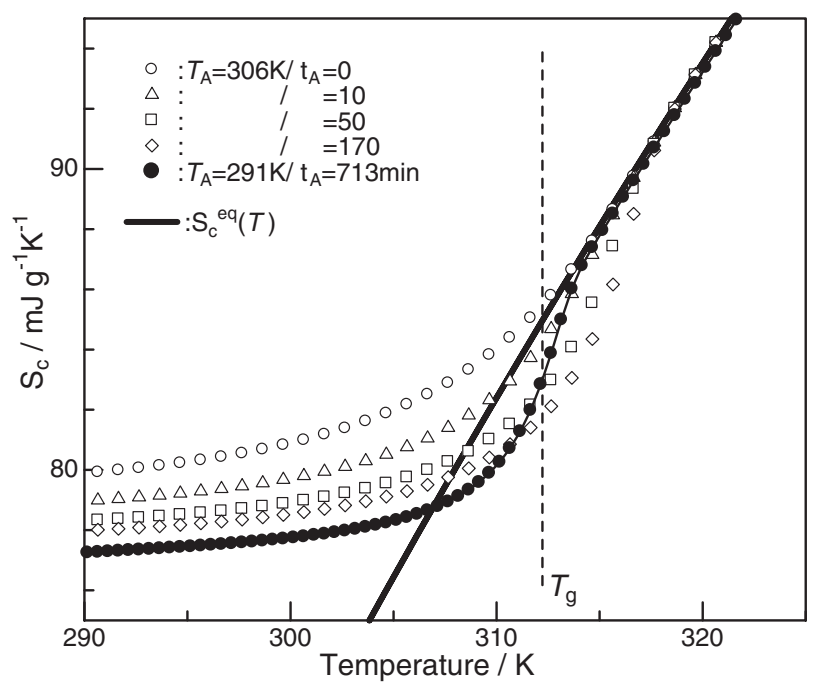

Figure 4. The relation between $S_{c}(t)$ and temperature for different isothermal ageings obtained by the calculation during the final heating scan. $B=1500 \mathrm{~J} / \mathrm{g}$ and the remaining parameters are shown in Table II. The solid line represents $S_{\mathrm{c}}{ }^{\mathrm{eq}}(T)$.

Figure 2 were omitted to prevent overlapping. Figure 4 contains $S_{\mathrm{c}}{ }^{\mathrm{eq}}(T)$ for the reference, which was calculated by eq 7 with $T_{2}$ shown in Table II. Diagrams such as Figure 4 is often used to explain the relaxation of the physical properties. In particular, the diagram of enthalpy-temperature curve is displayed in many literatures to illustrate enthalpic $T_{\mathrm{g}}$, unrelaxed enthalpy, equilibrium glassy state and so on. ${ }^{35-38}$ However, this paper is the first, to the author's knowledge, that $S_{\mathrm{c}}$ curve was constructed concerning the real material for the specified $T_{\mathrm{A}}$ and $t_{\mathrm{A}}$.

$S_{\mathrm{c}}(t)$ obtained in this work had roughly the same value as shown by Andreozzi et al., where the configurational entropy is discussed for a side-chain liquid crystalline polymer (The acronym is PMA4.) of $T_{\mathrm{g}}=292 \mathrm{~K} .{ }^{34} S_{\mathrm{c}}(t)$ of each aged sample shows the plateau value up to $T \approx 305 \mathrm{~K}$, then it increases with further rise in temperature. After passing $T_{\mathrm{g}}$ it continues to increase and finally meets up with the line of $S_{\mathrm{c}}{ }^{\mathrm{eq}}(T) . S_{\mathrm{c}}(t)$ of $t_{\mathrm{A}}=0$, i.e., the reference scan is higher than $S_{\mathrm{c}}{ }^{\mathrm{eq}}(T)$ until the meeting. While, $S_{\mathrm{c}}(t)$ of each ageing time once gets lower than $S_{\mathrm{c}}{ }^{\mathrm{eq}}(T)$ then increases rapidly and meets up with $S_{\mathrm{c}}{ }^{\mathrm{eq}}(T)$; the rapid increase corresponds with the recovery of entropy mentioned above. For plots of $T_{\mathrm{A}}=306 \mathrm{~K}$, the temperatures at which they meet shift higher as the increase in $t_{\mathrm{A}}$, which coincides with the shift of the peak position in Figure 2. It can be concluded that the behaviour of $S_{\mathrm{c}}(t)$ dominates the peak position. The peaks of $T_{\mathrm{A}}=306 / t_{\mathrm{A}}=10$ and $T_{\mathrm{A}}=291 / t_{\mathrm{A}}=$ 713, shown in Figures 2 and 3 respectively, can be taken as the example, where the meeting temperatures in Figure 4 are nearly equivalent and the peak positions are also same.

Minute investigations are required into the curve of $S_{\mathrm{c}}(t) v s$. temperature to grasp the details of the peak of overshoot such as the peak width, the peak height, the temperature of the onset and the maximum, and so on. ${ }^{39}$ In addition, the balance between heating and cooling rates is pointed out to influence 


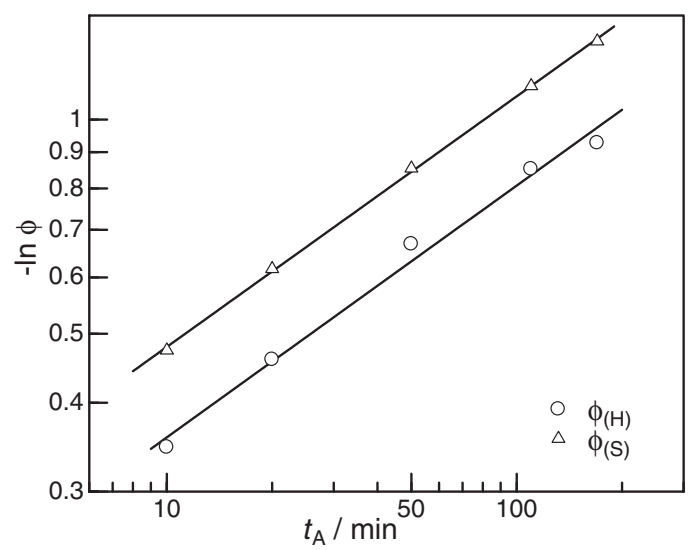

Figure 5. Double logarithmic plots of $-\ln \phi v s$. $t_{A}$ obtained for the isothermal ageing of PCBA6 at $T_{\mathrm{A}}=306 \mathrm{~K} . \phi_{(\mathrm{H})}$ is determined from $\Delta H\left(t_{\mathrm{A}}, T_{\mathrm{A}}\right)$ measured by DSC. $\phi_{(\mathrm{S})}$ is determined from $\Delta S\left(t_{\mathrm{A}}, T_{\mathrm{A}}\right)$ obtained by the calculation using $\mathrm{SC}$ model.

the peak position. However those investigations are out of scope in this paper.

Next, the $\delta$ parameter was introduced in SC model and the curve fitting was carried out with the same method described above. As a result, the $c_{\mathrm{p}}$ curve calculated with the $\delta$ parameter is not different from the curve calculated with $\delta=0$. That is, the introducing $\delta$ into $\Delta c_{\mathrm{p}}{ }^{\lim }(T)$ did not improve remarkably the difference of the peak height in $c_{\mathrm{p}}$ curve of the ageing time longer than $1000 \mathrm{~min}$ between the calculation and the experimental result shown in Figure 2. SC model analysis without using the $\delta$ parameter has also been reported for the three kinds of polymethacrylate. ${ }^{9}$ However, the introducing $\delta$ in SC model can be seen in almost all the modelling studies reported concerning the enthalpy relaxation of polymer. ${ }^{8,10,11,13-15,34}$

The proposed measure for the structural relaxation of PCBA6 is the value of $\phi$ as mentioned in the introduction section. $\phi$ determined through the configurational entropy, that is written as $\phi_{(\mathrm{S})}$, is defined as follows,

$$
\phi_{(\mathrm{S})}=\frac{\Delta S\left(\infty, T_{A}\right)-\Delta S\left(t_{\mathrm{A}}, T_{A}\right)}{\Delta S\left(\infty, T_{A}\right)} .
$$

The definition of eq 14 is similar to that of enthalpy measured by $\quad \mathrm{DSC} ; \quad \phi_{(\mathrm{H})}=\left[\Delta H\left(\infty, T_{\mathrm{A}}\right)-\Delta H\left(t_{\mathrm{A}}, T_{\mathrm{A}}\right)\right] / \Delta H\left(\infty, T_{\mathrm{A}}\right)$. $\Delta S\left(\infty, T_{\mathrm{A}}\right)$ is the asymptotic value of $\Delta S\left(t_{\mathrm{A}}, T_{\mathrm{A}}\right)$ and obtained by subtracting $S_{\mathrm{c}}{ }^{\mathrm{eq}}(T)$ from $S_{\mathrm{c}}(t)$ of the reference scan at $T_{\mathrm{A}}$. (see Figure 4$) \phi_{(\mathrm{S})}$ is therefore the departure from the equilibrium.

$\phi_{(\mathrm{S})}$ was evaluated for the thermal ageing at $T_{\mathrm{A}}=306 \mathrm{~K}$ based on the results of $10 \leq t_{\mathrm{A}} \leq 170$ shown in Figures 2 and 3. $\phi_{(\mathrm{H})}$ of previous results were also collected. Figure 5 shows $\phi_{(\mathrm{S})}$ and $\phi_{(\mathrm{H})}$ as a double logarithmic plot of $-\ln \phi v s$. $t_{\mathrm{A}}$. The straight line in the double logarithmic plot means the stretched exponential relation of eq $4 . \phi_{(\mathrm{S})}$ and $\phi_{(\mathrm{H})}$ had the same slope with the different $y$-intercept, showing that $\beta$ are same and $\tau$ are different. At first glance this result seems to disagree with the assumption of the same relaxation times given in eq 12. It is considered to come from the estimations of $\Delta S\left(\infty, T_{\mathrm{A}}\right)$ and $\Delta H\left(\infty, T_{\mathrm{A}}\right)$. That is, in determining $\Delta H\left(\infty, T_{\mathrm{A}}\right)$ the linear

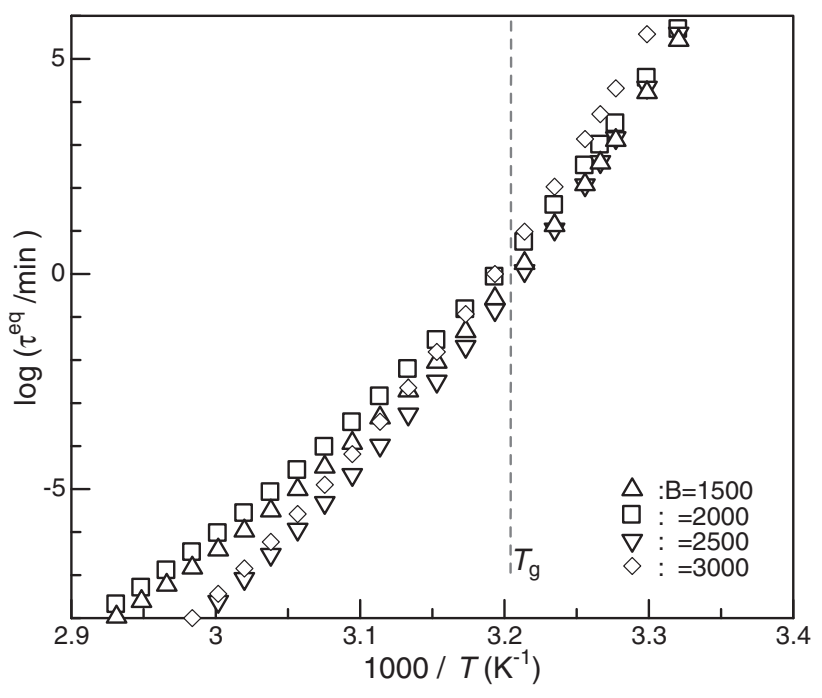

Figure 6. The relation between the equilibrium structural relaxation times and $(1000 / T)$ obtained using different sets of parameters of Table II. The coincidence of the different curves around $T_{\mathrm{g}}$ is remarkable.

relation between enthalpy and temperature was assumed and the equation of $\Delta H\left(\infty, T_{\mathrm{A}}\right)=\left(T_{\mathrm{g}}-T_{\mathrm{A}}\right) \times \Delta c_{\mathrm{p}}$ was used. Whereas $S_{\mathrm{c}}{ }^{\mathrm{eq}}(T)$ is used in determing $\Delta S\left(\infty, T_{\mathrm{A}}\right)$, strictly speaking, which does not show linear dependence on temperature.

The basic feature of $\phi_{(\mathrm{S})}$ and $\phi_{(\mathrm{H})}$ was described here. At first, both $\phi_{(\mathrm{S})}$ and $\phi_{(\mathrm{H})}$ are determined for the states of the start and the finish of the ageing. They are independent of further details of the temperature history. Second, they reflect the ageing conditions of both $t_{\mathrm{A}}$ and $T_{\mathrm{A}}$. Next, $\phi_{(\mathrm{H})}$ is determined by the experiment while $\phi_{(\mathrm{S})}$ is determined by the calculation; they have the correlation as shown in Figure 5. With these features, the values of $\phi_{(\mathrm{S})}$ and $\phi_{(\mathrm{H})}$ are considered to be suitable to measure the structural change of PCBA6 upon the ageing. Also $\phi$ has an unsuitable point; that is, it is impossible to compare $\phi$ values between different properties of different samples because $\phi$ is the fraction. For example, $\phi$ values cannot be compared directly between PCBA of different spacer lengths.

In order to lay more confidence on the parameters shown in Table II, they were investigated in terms of the relaxation mechanism characterised by the equilibrium relaxation time of eq 5. It was calculated from $S_{\mathrm{c}}{ }^{\mathrm{eq}}(T)$ and plotted in Figure 6 in order to verify whether or not the different sets of parameters uniquely determine $\tau^{\mathrm{eq}}(T)$. Values obtained for PCBA6 were in the same order as those reported for poly(butyl methacrylate) $\left(T_{\mathrm{g}}=310 \mathrm{~K}\right)$ and PMA4..$^{9,34}$ The curves are almost indistinguishable in the narrow temperature range around $T_{\mathrm{g}}$, which range is sensitive in calculating the $c_{\mathrm{p}}(T)$ curve.

Ribelles et al. regard these valuables as the material parameters in consequence that the unique temperature dependence of $\tau^{\mathrm{eq}}(T)$ has been determined from different sets of parameters. Concerning the existence of the material parameters, it cannot be mentioned clearly because the sample 
is limited. In particular, the introducing $\delta$ did not improve the $c_{\mathrm{p}}(T)$ curve remarkably in this work. It is thus necessary to find some complementary method to decide between the different sets of parameters.

\section{Concluding Remarks}

$c_{\mathrm{p}}(T)$ curves of DSC measurement for PCBA6 were reproduced by the calculation using SC model. In reproducing, different sets of parameters were determined. It was confirmed from the temperature dependence of $\tau^{\mathrm{eq}}(T)$ that the different sets lead to the unique relaxation mechanism. The evolution of out-of-equilibrium configurational entropy was shown to account for the peak position in the $c_{\mathrm{p}}(T)$ curve. In DSC heating scan, $S_{\mathrm{c}}(t)$ rapidly approaches to $S_{\mathrm{c}}{ }^{\mathrm{eq}}(T)$ according to the thermal history PCBA6 has experienced, where DSC peak appears. The values of $\phi_{(\mathrm{S})}$ and $\phi_{(\mathrm{H})}$ have the meaning of the measure of structural relaxation. $\phi_{(\mathrm{S})}$ is determined from the calculation and $\phi_{(\mathrm{H})}$ from the DSC measurement; they are not independent.

Received: September 20, 2008

Accepted: October 24, 2008

Published: December 11, 2008

\section{REFERENCES}

1. Y. Tanaka, S. Takahashi, R. Kishi, and H. Ichijo, Mol. Cryst. Liq. Cryst., 339, 45 (2000).

2. Y. Tanaka, R. Kishi, and H. Ichijo, Mol. Cryst. Liq. Cryst., 348, 179 (2000).

3. Y. Tanaka, Polym. J., 39, 1030 (2007).

4. A. Q. Tool, J. Am. Ceram. Soc., 29, 240 (1946).

5. G. W. Scherer, J. Am. Ceram. Soc., 67, 504 (1984).

6. I. M. Hodge and A. R. Berens, Macromolecules, 14, 1598 (1981).

7. I. M. Hodge, Macromolecules, 20, 2897 (1987).

8. J. L. Gomez Ribelles and M. Monleon Pradas, Macromolecules, 28, 5867 (1995).

9. J. L. Gomez Ribelles, M. Monleon Pradas, A. Vidaurre Garayo, F. Romero Colomer, J. Mas Estelles, and J. M. Meseguer Duenas, Macromolecules, 28, 5878 (1995).

10. J. L. Gomez Ribelles, M. Monleon Pradas, A. Vidaurre Garayo, F. Romero Colomer, J. Mas Estelles, and J. M. Meseguer Duenas, Polymer, 38, 963 (1997).

11. J. M. Meseguer Duenas, A. Vidaurre Garayo, F. Romero Colomer,
J. Mas Estelles, J. L. Gomez Ribelles, and M. Monleon Pradas, J. Polym. Sci., B: Polym. Phys., 35, 2201 (1997).

12. J. L. Gomez Ribelles, J. M. Meseguer Duenas, C. Torregrosa Cabanilles, and M. Monleon Pradas, J. Phys.: Condens. Matter, 15, S1149 (2003).

13. N. M. Alves, J. L. Gomez Ribelles, and J. F. Mano, Polymer, 46, 491 (2005).

14. L. Andreozzi, M. Faetti, F. Zulli, and M. Giordano, Eur. Phys. J. B, 41, 383 (2004).

15. L. Andreozzi, M. Faetti, F. Zulli, and M. Giordano, Macromolecules, 37, 8010 (2004).

16. L. Andreozzi, M. Faetti, M. Giordano, D. Palazzuoli, and F. Zulli, Macromolecules, 36, 7379 (2003).

17. C. T. Moynihan, A. J. Easteal, D. C. Tran, J. A. Wilder, and E. P. Donovan, J. Am. Ceram. Soc., 59, 137 (1976).

18. I. M. Hodge, Macromolecules, 16, 898 (1983).

19. T. Nagaya, H. Hotta, H. Orihara, and Y. Ishibashi, J. Phys. Soc. Jpn., 61, 3511 (1992).

20. C. T. Moynihan, A. J. Easteal, M. A. DeBolt, and J. Tucker, J. Am. Ceram. Soc., 59, 12 (1976).

21. M. A. DeBolt, A. J. Easteal, P. B. Macedo, and C. T. Moynihan, J. Am. Ceram. Soc., 59, 16 (1976).

22. G. Williams and D. C. Watts, Trans. Faraday Soc., 66, 80 (1970).

23. G. Adam and J. H. Gibbs, J. Chem. Phys., 43, 139 (1965).

24. O. S. Narayanaswamy, J. Am. Ceram. Soc., 54, 491 (1971).

25. J. H. Gibbs and E. A. DiMarzio, J. Chem. Phys., 28, 373 (1958).

26. T. I. Gubina, S. Kise, S. G. Kostromin, R. V. Talrose, V. P. Shivaev, and N. A. Platé, Liq. Cryst., 4, 197 (1989).

27. Concerning DSC apparatus, the incorrect model number was described in the previous paper of ref 3. The correct number is SEIKO DSC 200.

28. J. L. Gomez Ribelles, A. Ribes Greus, and R. Diaz Calleja, Polymer, 31, 223 (1990).

29. J. J. Tribone, J. M O'Reilly, and J. Greener, Macromolecules, 19, 1732 (1986).

30. I. M. Hodge and A. R. Berens, Macromolecules, 15, 762 (1982).

31. I. M. Hodge and G. S. Huvard, Macromolecules, 16, 371 (1983).

32. F. Romero Colomer and J. L. Gomez Ribelles, Polymer, 30, 849 (1989).

33. C. Alvarez, N. T. Correia, J. J. Moura Ramos, and A. C. Fernandes, Polymer, 41, 2907 (2000).

34. L. Andreozzi, M. Faetti, M. Giordano, and D. Palazzuoli, Macromolecules, 35, 9049 (2002).

35. M. J. Richardson and N. G. Savill, Polymer, 16, 753 (1975).

36. A. S. Marshall and S. E. B. Petrie, J. Appl. Phys., 46, 4223 (1975).

37. A. R. Berens and I. M. Hodge, Macromolecules, 15, 756 (1982).

38. J. M. G. Cowie and R. Ferguson, Macromolecules, 22, 2307 (1989).

39. D. Lacey, G. Nestor, and M. J. Richardson, Thermochim. Acta, 238, 99 (1994). 